\title{
Early methyl donor deficiency may induce persistent brain defects by reducing Stat3 signaling targeted by miR-124
}

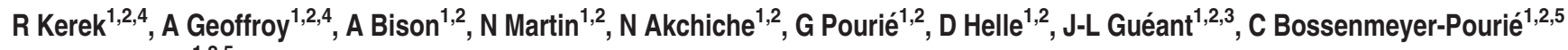 \\ and J-L Daval ${ }^{*, 1,2,5}$
}

The methyl donors folate (vitamin B9) and vitamin B12 are centrepieces of the one-carbon metabolism that has a key role in transmethylation reactions, and thus in epigenetic and epigenomic regulations. Low dietary intakes of folate and vitamin B12 are frequent, especially in pregnant women and in the elderly, and deficiency constitutes a risk factor for various diseases, including neurological and developmental disorders. In this respect, both vitamins are essential for normal brain development, and have a role in neuroplasticity and in the maintenance of neuronal integrity. The consequences of a methyl donor deficiency (MDD) were studied both in vivo in rats exposed in utero, and in vitro in hippocampal progenitors (H19-7 cell line). Deficiency was associated with growth retardation at embryonic day 20 (E20) and postnatally with long-term brain defects in selective areas. mRNA and protein levels of the transcription factor Stat3 were found to be decreased in the brains of deprived fetuses and in differentiating progenitors ( 62 and $48 \%$ for total Stat3 protein, respectively), along with a strong reduction in its phosphorylation at both $\mathrm{Tyr}^{705}$ and $\mathrm{Ser}^{727}$ residues. Vitamin shortage also affected upstream kinases of Stat3 signaling pathway (phospho-Erk1/2, phospho-Src, phospho-JNK, and phospho-p38) as well as downstream target gene products (Bcl-2 and Bcl-xL), thus promoting apoptosis. Conversely, the expression of the Stat3 regulator miR-124 was upregulated in deficiency conditions ( $\geq 65 \%)$, and its silencing by using siRNA partly restored Stat3 signaling in hippocampal neurons by increasing specifically the phosphorylation of Erk1/2 and Src kinases. Furthermore, miR-124 siRNA improved the phenotype of deprived cells, with enhanced neurite outgrowth. Taken together, our data suggest that downregulation of Stat3 signaling by miR-124 would be a key factor in the deleterious effects of MDD on brain development.

Cell Death and Disease (2013) 4, e755; doi:10.1038/cddis.2013.278; published online 8 August 2013

Subject Category: Neuroscience

The link between diet during pregnancy and infant development has been largely documented, and was associated with long-term effects on health. ${ }^{1-3}$ The prevalence of folate (vitamin B9) and/or cobalamin (vitamin B12) deficiency in pregnant women is relatively high, especially in some communities. ${ }^{4,5}$ The one-carbon metabolism is essential for DNA biosynthesis and methylation reactions, and thus has a key role in epigenetic and epigenomic regulations. ${ }^{6}$ The conversion of 5,10-methylenetetrahydrofolate to tetrahydrofolate in the folate cycle provides the methyl group required to convert the sulphur-containing amino acid homocysteine to methionine. Vitamin B12 acts as a cofactor for methionine synthase, which catalyzes this reaction. ${ }^{7}$ Methionine is the precursor of S-adenosylmethionine (SAM), the universal substrate of transmethylation reactions, which is then demethylated to form S-adenosylhomocysteine (SAH). Thereafter, homocysteine is generated from the hydrolysis of SAH. The shared metabolism between folate and vitamin B12 suggests that deficiencies in one vitamin may alter the metabolism of the other. Deficiency of either folate, vitamin B12 or both vitamins can lead to various adverse consequences, including elevated blood homocysteine, intrauterine growth retardation, congenital heart defects and Down's syndrome..$^{8,9}$ Importantly, folate and vitamin B12 are essential for normal brain development, and both reduced folate and vitamin B12 status are risk factors for developmental disorders such as neural tube defects that can originate at various times during gestation. ${ }^{10}$ In addition, a large body of evidence has linked deficiencies in maternal folate and/or vitamin B12 with infant cognitive development. 8,9

\footnotetext{
${ }^{1}$ Faculté de Médecine, Inserm U954, Vandoeuvre-lès-Nancy, France; ${ }^{2}$ Faculté de Médecine de Nancy, Université de Lorraine, Vandoeuvre-lès-Nancy, France and ${ }^{3}$ IRCCS, Oasi Maria S.S., Institute for Research on Mental Retardation and Brain Aging, Troina, Italy

*Corresponding author: J-L Daval, Inserm U954, Faculté de Médecine, 9 Avenue de la Forêt de Haye, Vandoeuvre-lès-Nancy F-54500, France. Tel: + 333836832 82; Fax: + 3338368 32 79; E-mail: jean-luc.daval@inserm.fr

${ }^{4}$ These authors countributed equally to this work.

${ }^{5}$ These authors countributed equally to this work.

Keywords: folate; vitamin B12; Stat3; miR-124; epigenetics; brain development

Abbreviations: Dapi, 4,6-diamidino-2-phenylindole; DMEM, Dulbecco's modified Eagle's medium; E, embryonic day; Erk, extracellular signal-regulated kinase; GAPDH, Glyceraldehyde-3-phosphate dehydrogenase; JNK, c-Jun N-terminal kinase; MAP, mitogen-activated protein; MDD, methyl donor deficiency; PN, postnatal day; RT-qPCR, reverse transcription quantitative polymerase chain reaction; SAH, S-adenosylhomocysteine; SAM, S-adenosylmethionine; siRNA, small interfering RNA; Stat3, signal transducer and activator of transcription 3; SVZ, subventricular zone; TUNEL, Terminal deoxynucleotidyl transferase dUTP nick end labeling Received 19.4.13; revised 25.6.13; accepted 02.7.13; Edited by E Candi
} 
In experimental studies, deficiency in methyl donors such as folate and B12 has been associated with imbalanced proliferation/differentiation of neuronal cells, endoplasmic reticulum stress and apoptosis. ${ }^{11-15}$ In addition, hippocampus atrophy, rarefaction of microvasculature as well as cognitive impairments were reported in rodents. ${ }^{12,16,17}$ Although homocysteine was shown to be embryotoxic, it did not appear to be directly responsible for developmental disorders, ${ }^{18,19}$ and the mechanisms underlying the effect of maternal B vitamin status on neural tube and brain development remain poorly understood.

Embryofetal brain development is a very complex process, requiring the temporal expression of specific gene sets with a number of precisely orchestrated steps. ${ }^{20}$ To determine how genetic regulation of factors related to neural development might account for brain defects associated with folate/vitamin B12 depletion, we used a microarray approach (Rat Neurogenesis and Neural Stem Cell PCR Array, $\mathrm{RT}^{2}$ Profiler PCR Array, SABiosciences, Qiagen SA, Courtaboeuf, France) that profiled the expression of 84 genes related to neurogenesis and cell differentiation in hippocampal neuroprogenitors issued from the rat $\mathrm{H} 19-7$ hippocampal embryonic cell line and in the brain of rat pups subjected to early methyl donor deficiency (MDD) (unpublished observations). As signal transducer and activator of transcription 3 (Stat3) emerged as one gene highly downregulated upon vitamin B deficiency, we further investigated its participation in the consequences of MDD both in vivo on a validated rat model of maternal deficiency ${ }^{12,17}$ and in vitro in hippocampal progenitors. ${ }^{14}$

Primarily associated with cytokine- and growth factorrelated signals, Stat3 is activated through phosphorylation, and regulates the transcription of target genes that participate in a number of physiological responses. ${ }^{21}$ Indeed, Stat3 has been repeatedly involved in fundamental biological processes, such as cell proliferation, differentiation, apoptosis and survival, ${ }^{22,23}$ and has been shown to be implicated in various developmental events, such as motor neuron pathfinding and guidance ${ }^{24}$ and neurite outgrowth. ${ }^{25}$ Here, we provide the first evidence that early MDD causes loss of Stat3 signaling related to overexpression of $\mathrm{miR}-124$, with impairment of cerebral development.

\section{Results}

In vivo and in vitro earlier studies have demonstrated the efficiency of our experimental conditions in reducing methyl donor availability both in the rat progeny ${ }^{12,26}$ and in H19-7 hippocampal cells. ${ }^{14}$ Consistently, global DNA methylation was reduced in low methyl donor conditions in the two experimental models, that is, by $23.2 \%$ in the brain tissue from fetuses $(n=4)$ and by $40.4 \%$ in cultured hippocampal cells at $13 \mathrm{~h}$ after induction of their differentiation $(n=4)$.

Gestational MDD alters rat pup morphometric properties and brain characteristics. As shown by Figure 1, both body weight and body length were significantly reduced ( $-20.5 \%$ and $-15 \%$, respectively) during late gestation (embryonic day 20, E20) in rat fetuses from dams fed the deficient diet, suggesting the occurrence of intrauterine growth retardation. This was confirmed by the noticeable reduction in the femur length $(-14 \%)$. Whereas brain weight was also affected ( $-14.6 \%)$, the thickness of selective brain layers such as the hippocampal CA1 pyramidal layer, the granular cell layer of the dentate gyrus and the neurogenic subventricular zone (SVZ) were more dramatically decreased $(-40,-22$ and $-26 \%$, respectively). Most interestingly, these brain areas remained atrophied as late as the age of 450 days, although rat pups had exclusively received a standard, non-deficient diet since their weaning (Figures 1e-h).

MDD decreases brain expression and phosphorylation of Stat3. When studied by quantitative RT-PCR analysis, mRNA levels of Stat3 were found to be significantly decreased in the brains of E20 fetuses subjected to MDD as compared with controls ( $-34 \%$, Figure $2 a$ ). Consistently, western blot immunoactivities revealed a decreased expression of Stat3 total protein both in the midbrain (which includes hippocampus, thalamus, hypothalamus and striatum) and in the cerebral cortex, along with lowered amounts of the phosphorylated forms, that is, phospho-Stat3 $\mathrm{Tyr}^{705}$ and phospho-Stat3 Ser $^{727}$ (Figure 2b). Quantitative changes in protein levels are provided in Supplementary information (Supplementary Figure 1). According to our western blot analyses, total amounts of Stat3 protein were reduced by $62 \%$ in the midbrain of deprived fetuses. As illustrated by Figure 2c for phospho-Stat3 $\mathrm{Tyr}^{705}$, such observations were confirmed in situ by immunohistochemistry in the cerebral cortex, the SVZ and the CA1 layer of the hippocampus.

Similar findings were obtained in cultured hippocampal progenitor cells, with a decrease in mRNA levels ( $-54 \%$ ) and protein expression at 6 and $13 \mathrm{~h}$ after the induction of differentiation in cells lacking folate (Figure 3 and Supplementary Figure 1). Amounts of total Stat3 protein were reduced by $48 \%$ at $13 \mathrm{~h}$ after induction of cell differentiation. In addition, immunocytochemical analyses by confocal microscopy revealed an obvious decrease in the presence of Stat3 in cell nuclei (Figure 3c).

MDD affects the expression of active key protein kinases. Stat3-signaling pathways require several kinases such as Erk1/2 MAP kinases, p38, c-Jun N-terminal kinase (JNK) and Src, which are involved in Stat3 dimerization and activation. ${ }^{27,28}$

Following exposure to MDD, the expression patterns of the active, phosphorylated forms of the corresponding kinases were differentially affected (Figure 4). Expression levels of phopho-Erk1/2, phospho-Src and phospho-p38 were significantly decreased, whereas the expression of phospho-JNK was increased and that of phospho-Akt did not vary significantly. Importantly, the recorded profiles were similar in the fetal brain tissues and in differentiating H19-7 cells. Quantitative changes in protein amounts are provided in Supplementary information (Supplementary Figure 2).

Decreased Stat3 signaling is associated with elevation of apoptosis and concomitant altered expression of related proteins both in vivo and in vitro. Activation of Stat3 is known to positively influence the expression of anti-apoptotic and pro-survival proteins $\mathrm{Bcl}-2$ and $\mathrm{Bcl}-\mathrm{xL} .{ }^{29,30}$ We therefore investigated their expression levels as well as those of other key factors in apoptosis following MDD. 

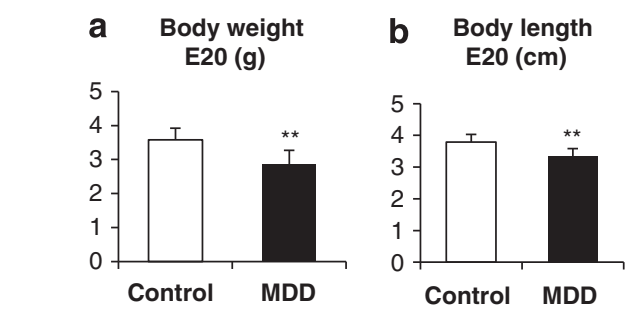

C Femur length

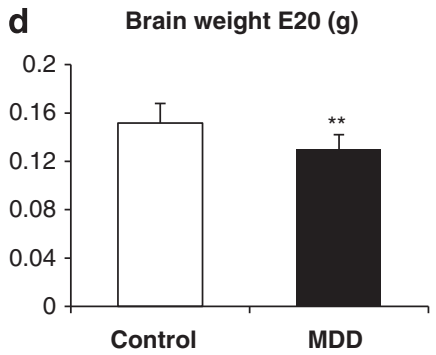

e

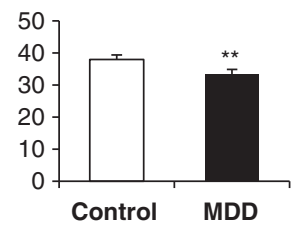

CA1 layer $(\mu \mathrm{m})$
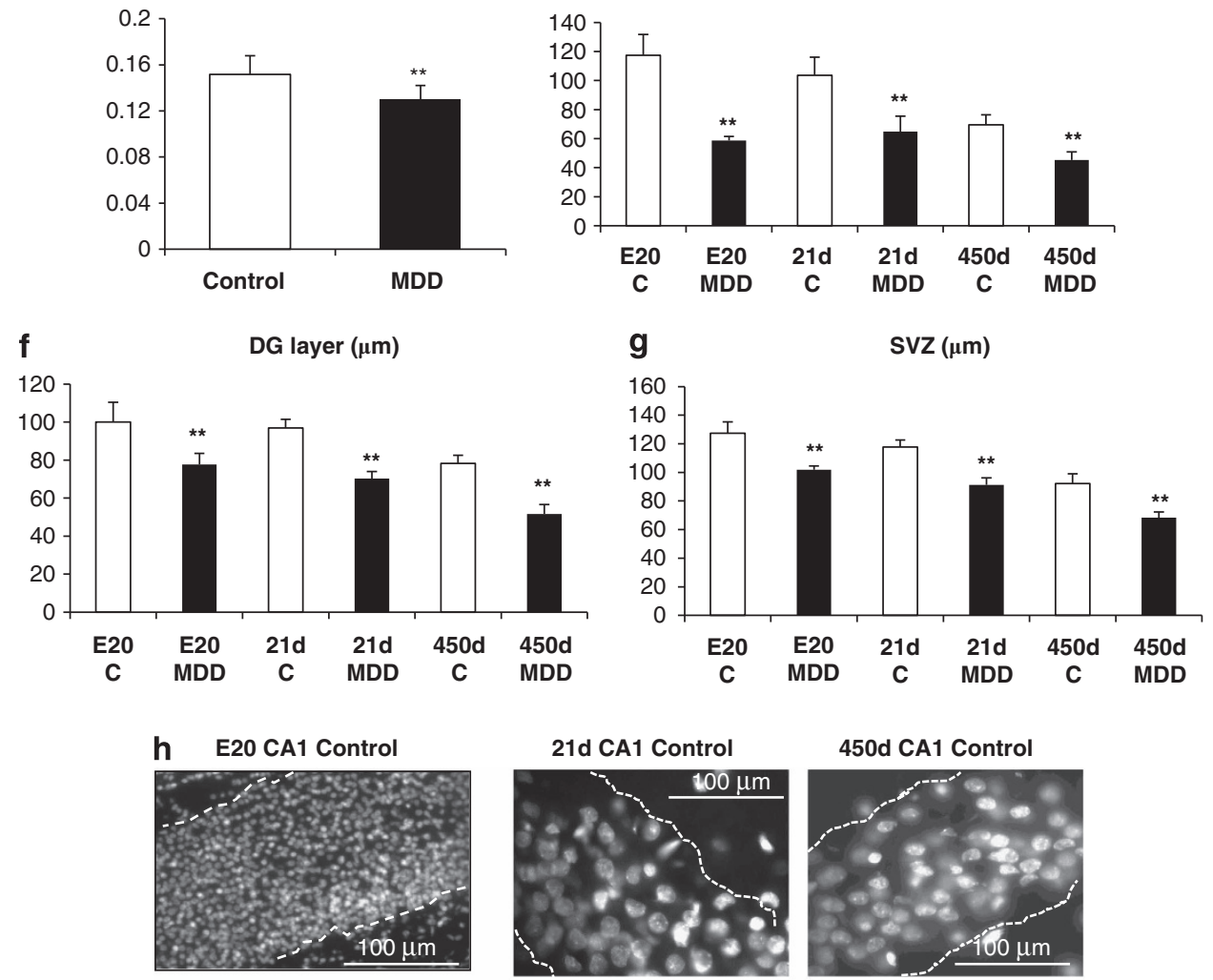

450d CA1 Control
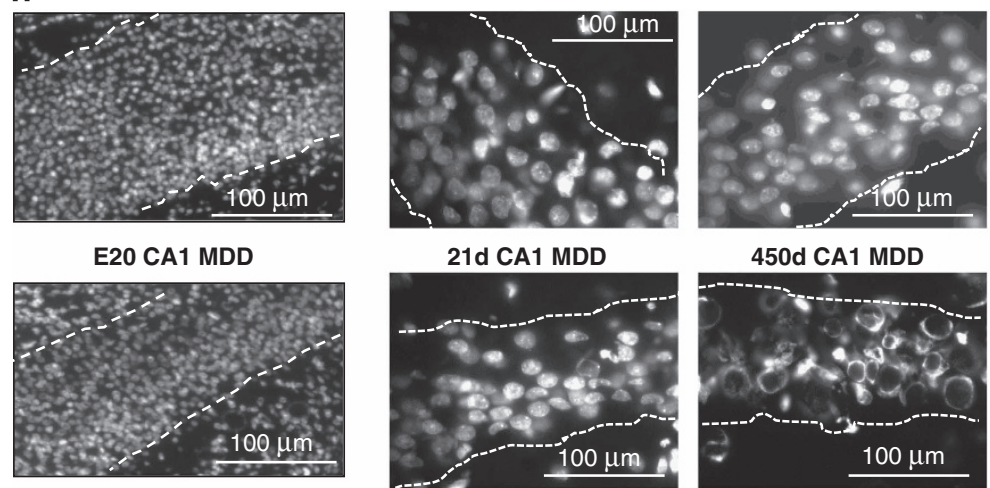

450d CA1 MDD

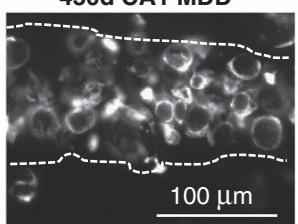

Figure 1 Effects of methyl donor deficiency on rat fetus morphometric properties. (a-d) General morphometric measurements in control and MDD fetuses at embryonic day (ED) 20,33 $\leq n \leq 37$. Data are reported as means \pm S.D. Statistically significant differences between control and MDD rats: ${ }^{* \star} P<0.01$. (e-g) Kinetic measurements of thicknesses of hippocampal CA1 pyramidal cell layer, granular cell layer of the dentate gyrus (DG), and SVZ at E20, 21 days (d) and 450 days of age, $n \geq 17$ and $n \geq 23$ for controls $(\mathrm{C})$ and $\mathrm{MDD}$, respectively, ${ }^{\star *} P<0.01$. (h) Histological illustrations of the effects of methyl donor deficiency in the CA1 pyramidal layer at the various ages after counterstaining of cell nuclei by DAPI

As expected, amounts of $\mathrm{Bcl}-2$ and $\mathrm{Bcl}-\mathrm{xL}$ were significantly decreased in the brain of E20 fetuses subjected to the deficient diet, whereas the expression levels of pro-apoptotic proteins Bax and cleaved caspase- 3 were more elevated in both midbrain and cerebral cortex, cleaved caspase-9 being overexpressed in the midbrain only (Figure 5a). Quantitative changes in protein amounts are provided in Supplementary information (Supplementary Figure 2). Furthermore, the number of TUNEL-positive cells was dramatically increased in the hippocampus, the SVZ and the cerebral cortex of deficient fetuses (Figure 5c, Supplementary Figure 3). This was confirmed by the labeling of active caspase- 3 and caspase- 9 in fetal brains, as illustrated in the CA1 hippocampal region and in the SVZ, with the presence of a high number of dead cells within the lateral ventricle (Supplementary Figure 3).

Similar findings were obtained from differentiating hippocampal progenitors, the viability of which was more affected 

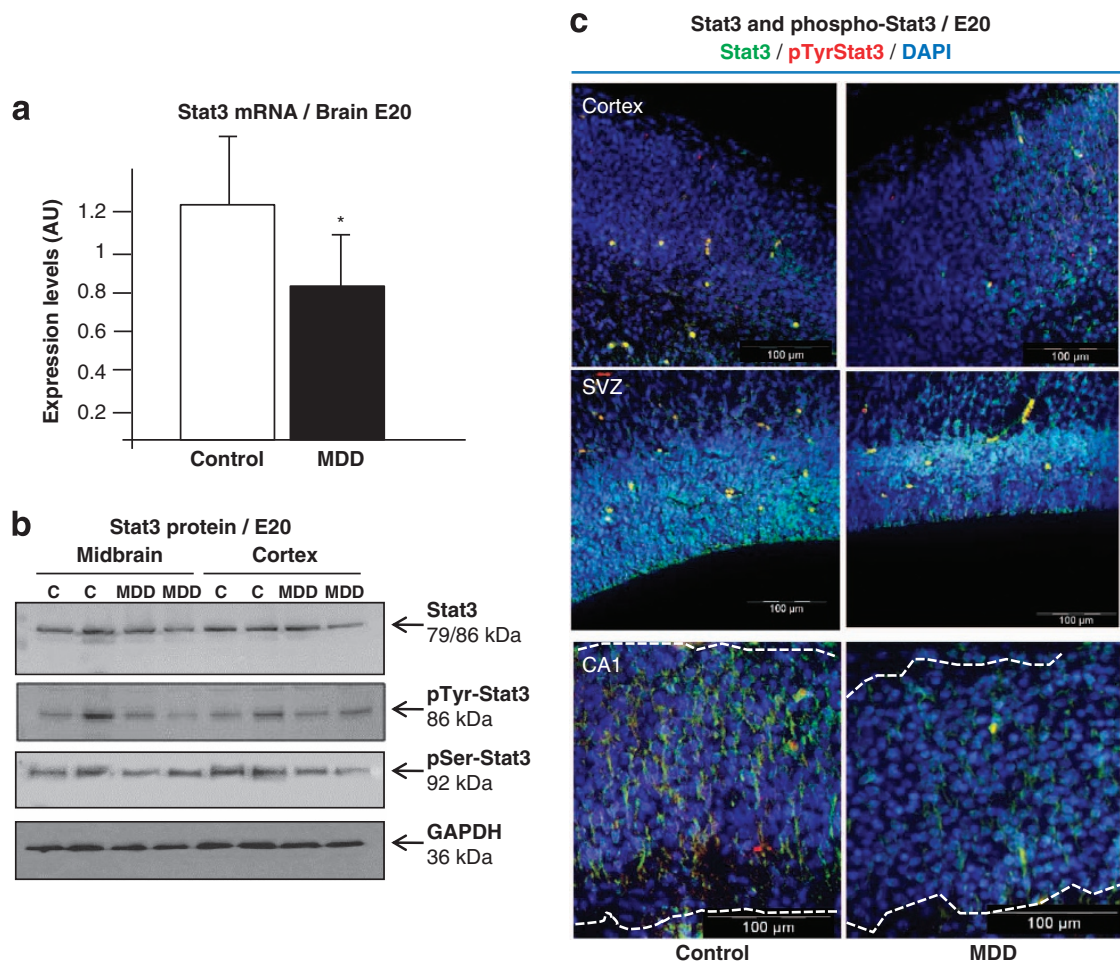

Figure 2 Effects of methyl donor deficiency on brain expression of Stat3 and phospho-Stat3 $\left(\operatorname{Tyr}^{705}\right.$ and Ser $\left.{ }^{727}\right)$ in rat fetuses at E20. (a) Total Stat3 mRNA levels measured by real-time quantitative RT-PCR. Data are reported in arbitrary units (AU) as means \pm S.D. $(n=5)$, ${ }^{*} P<0.05$. (b) Western blot analyses of protein expression levels of Stat3, phospho-Stat3 $\mathrm{Tyr}^{705}$ and phospho-Stat3 $\mathrm{Ser}^{727}$ in the midbrain and the cerebral cortex from control (C) and MDD E20 fetuses. Similar profiles were obtained from five other experiments. (c) Immunohistochemical labeling of Stat3 and phospho-Stat3 Tyr ${ }^{705}$ in the cerebral cortex, SVZ and hippocampal CA1 layer from control and MDD E20 fetuses

by folate deficiency as compared with controls (Figure $5 \mathrm{~d}$ ). Consistently, the loss of mitochondrial membrane potential, as depicted by the Mitocapture procedure, was higher in folate-deficient progenitors, along with an increased percentage of Apostain-positive cells indicative of apoptosisassociated single-stranded DNA (Figures 5e,f). Increased apoptosis was confirmed by the TUNEL/Apoptag method, as illustrated in Figure 5b.

Decreased Stat3 signaling is associated with increased brain expression of miR-124. As miR-124 has been reported to regulate Stat3 signaling by affecting Stat3 phosphorylation $^{31}$ and by targeting the $3^{\prime}$-UTR of Stat3 gene so as to inhibit the expression of Stat3 protein, ${ }^{32}$ we subsequently investigated the consequences of an MDD on the expression of miR-124 in the brain of rat pups and in H19-7 cells.

When studied using TaqMan RT-qPCR, miR-124a ncRNA expression patterns showed a significant increase (by twofold) in the brains from E20 fetuses exposed to MDD as well as in B9-deficient H19-7 neuroprogenitors at $13 \mathrm{~h}$ after the induction of their differentiation (1.7-fold) (Figures 6a,b). This was confirmed by in situ hybridization both in vivo in various brain areas including the hippocampus, the SVZ (Figure 6c) and the cerebellum (not shown), and in vitro in differentiating cells (Figure 6d).
Silencing miR-124 improves Stat3 signaling and at least partly rescues cells exposed to folate deficiency. The use of non-targeting siRNA had no patent effect on the expression of Stat3 at $13 \mathrm{~h}$ after induction of differentiation of H19-7 cells. Conversely, miR-124a siRNA, which repeatedly inhibited the expression of miR-124 by $87 \%$ after quantification by TaqMan RT-qPCR, increased Stat3 and phosphoStat3 levels, mainly in folate-deficient cells (Figure 7a). Consistently, silencing miR-124 obviously reduced the differences observed between control and deprived cells in the phosphorylation patterns of upstream Erk1/2 MAP and Src kinases, as well as in the expression of downstream targets of Stat3 (Bcl-2, Bcl-xL). Concomitantly, the amounts of Bax as well as active caspases 3 and 9 were found to be diminished in miR-124 siRNA-treated deprived cells, along with a significant decrease in TUNEL-positive cells (Figure 7c). Quantitative changes in protein amounts are provided in Supplementary information (Supplementary Figure 4). Silencing of miR-124 did not induce changes regarding the other kinases implicated in the Stat3 signaling pathway, that is, p38, JNK and Akt (data not shown).

The detrimental role of increased miR-124 in folatedeficient progenitors was further demonstrated by cell morphology of differentiating progenitors (Figure 7b). Indeed, folate deficiency was accompanied at $13 \mathrm{~h}$ of differentiation by a strong reduction in the number and length of neural 

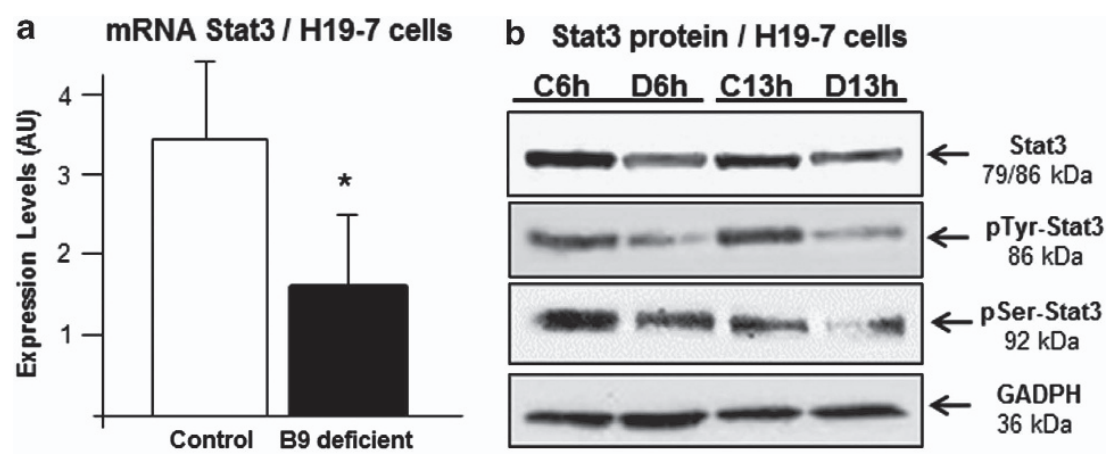

C Stat3 and phospho-Stat3 / H19-7 cells Stat3 / p TyrStat3 / DAPI Stat3 / pSerStat3 / DAPI
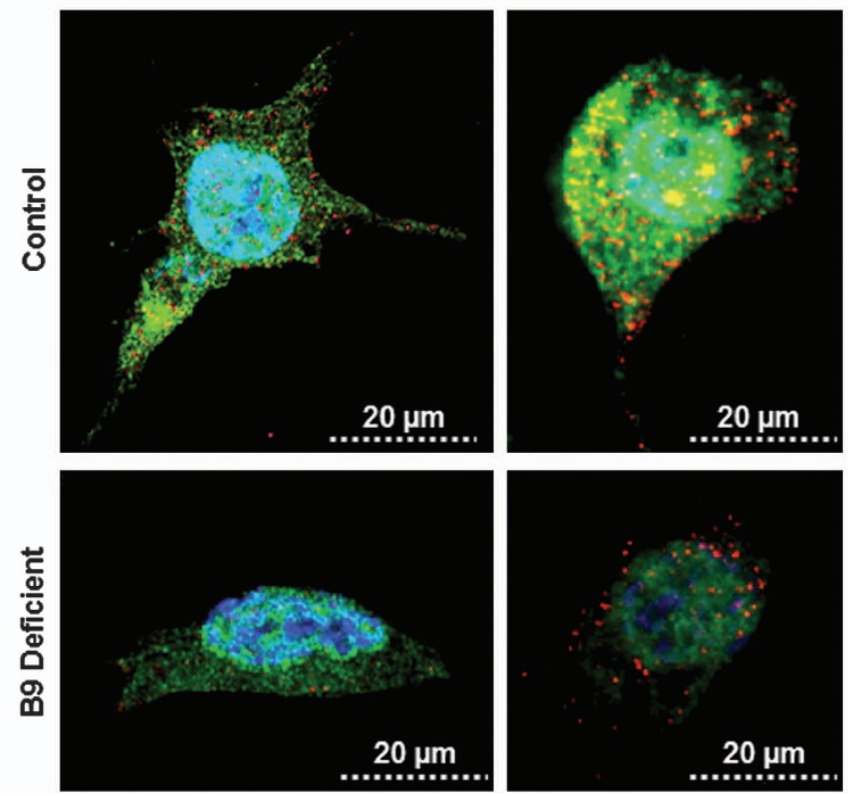

Figure 3 Effects of folate deficiency on the expression of Stat3 and phospho-Stat3 $\left(\mathrm{Tyr}^{705}\right.$ and $\mathrm{Ser}^{727}$ ) in $\mathrm{H} 19-7$ hippocampal progenitor cells. (a) Total Stat3 mRNA levels measured by real-time quantitative RT-PCR in control and B9-deficient cells at $13 \mathrm{~h}$ after induction of their differentiation. Data are reported in AUs as means \pm S.D. $(n=5)$, ${ }^{*} P<0.05$. (b) Western blot analyses of protein expression levels of Stat3, phospho-Stat3 $\mathrm{Tyr}^{705}$ and phospho-Stat3 $\mathrm{Ser}^{727}$ in control (C) and B9-deficient (D) H19-7 cells at 6 and $13 \mathrm{~h}$ after the induction of differentiation. Similar findings were obtained from five other experiments. (c) Immunocytochemical labeling of Stat3, phospho-Stat3 Tyr ${ }^{705}$ and phospho-Stat3 $\mathrm{Ser}^{727}$ in control and B9-deficient H19-7 cells at $13 \mathrm{~h}$ after induction of their differentiation

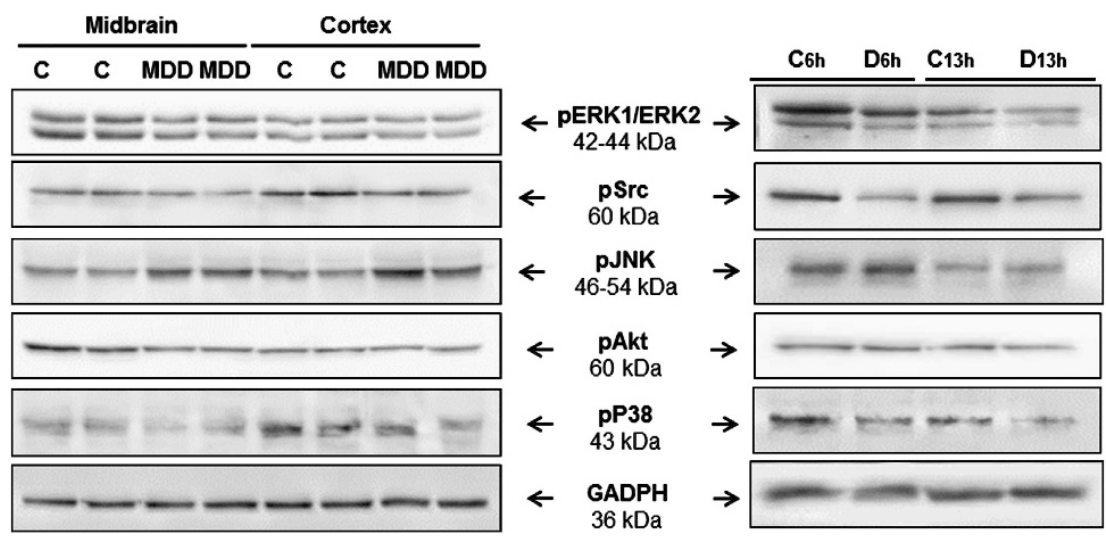

Figure 4 Western blot analyses of upstream phospho-kinases involved in Stat3 signaling pathways in the midbrain and cerebral cortex of control (C) and MDD E20 fetuses, and in control (C) and B9-deficient (D) H19-7 cells at 6 and13 h after the induction of their differentiation. Similar profiles were obtained from five other experiments 
a
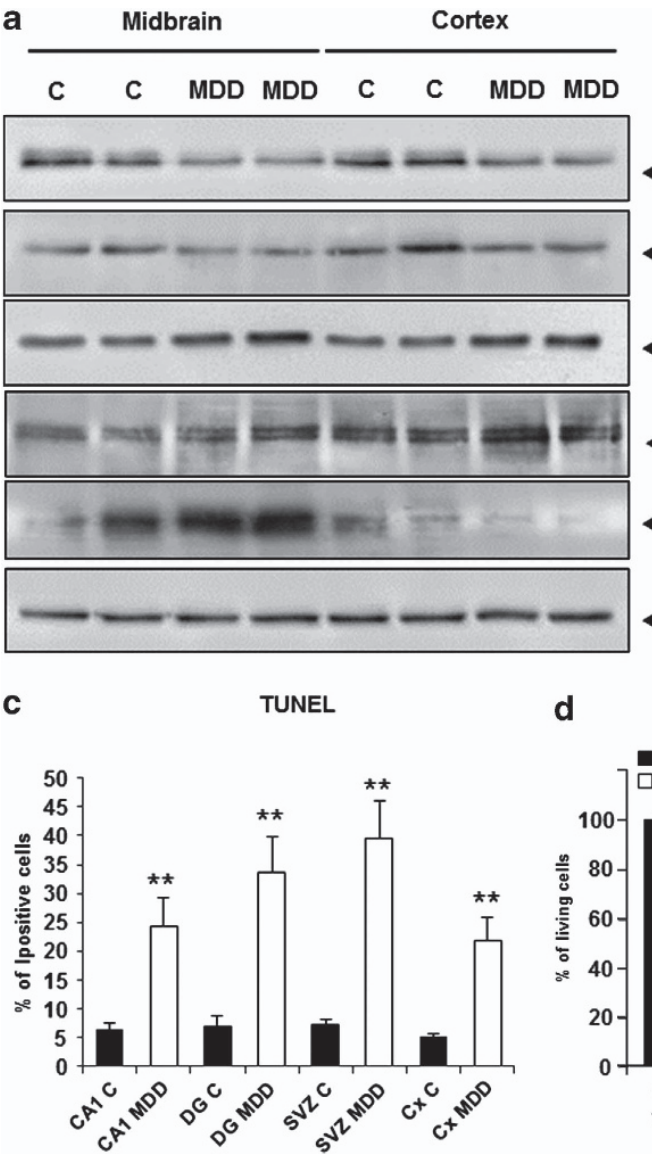

d

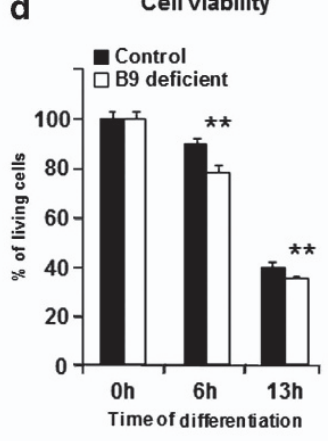

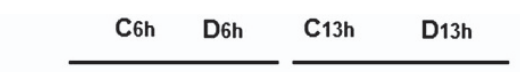

b

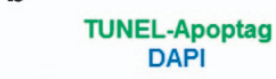

$\leftarrow \begin{array}{r}\mathrm{BclxL} \\ 30 \mathrm{kDa}\end{array}$
$\leftarrow \begin{array}{r}\mathrm{Bcl} 2 \\ 26 \mathrm{kDa}\end{array}$
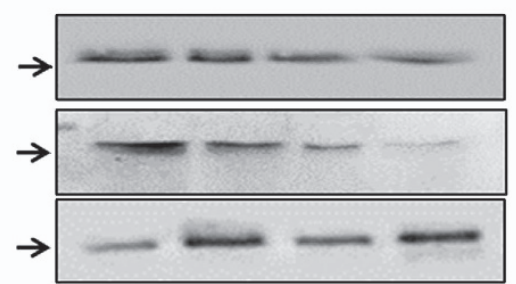

$\frac{5}{0}$
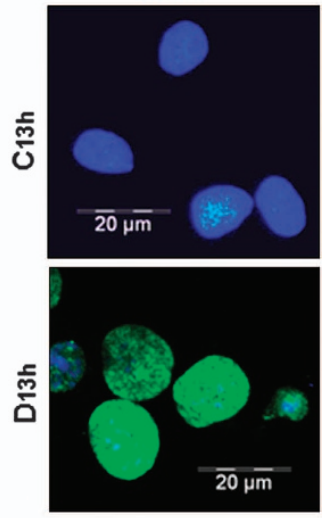

e

Mitocapture

f

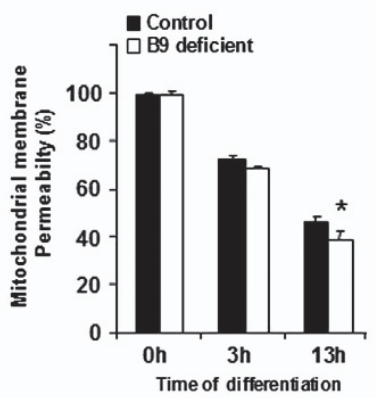

Apostain

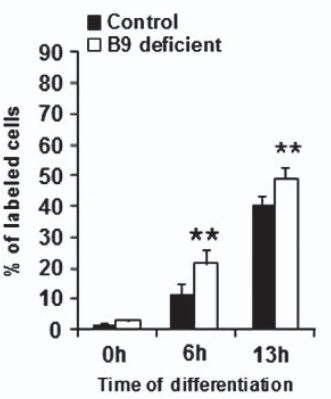

Figure 5 Effects of methyl donor deficiency on cell apoptosis and the key related proteins. (a) Western blot analyses of targets of Stat3 signaling (Bcl-xL and Bcl-2) and apoptosis-related proteins in the midbrain and cerebral cortex of control (C) and MDD E2O fetuses, and in control (C) and B9-deficient (D) H19-7 cells at 6 and13 h after the induction of their differentiation. Similar profiles were obtained from five separate experiments. (b) Illustration of increased apoptosis depicted by the TUNEL assay in deficient (D) cells as compared with controls (C) at $13 \mathrm{~h}$ following the induction of differentiation. (c) In situ quantification of apoptosis in the CA1 layer of the hippocampus, the DG, the SVZ and the cerebral cortex (Cx) in control (C) and MDD E20 fetuses. (d) Temporal changes in H19-7 cell viability by using the CellTiter-Glo luminescent cell viability assay (Promega). (e) Changes in the mitochondrial membrane potential depicted using the MitoCapture detection kit (Calbiochem). (f) Percent of apoptotic cells reflected by Apostain immunostaining. All data are reported as means \pm S.D. $(n=5) ;{ }^{*} P<0.05,{ }^{* *} P<0.01$

processes, as previously documented..$^{14}$ In the present study, the downregulation of miR-124 by siRNA led to a noticeable restoration of these processes. Although the treatment by miR-124 siRNA slightly affected cell characteristics in control progenitors, the number of neurites per cell was $\sim$ three-fold increased in folate-deficient cells treated by miR-124 siRNA as compared with those treated by non-targeting siRNA (Figures $7 \mathrm{~d}, P<0.01$ ).

\section{Discussion}

In the present study, we report for the first time that the downregulation of Stat3 signaling pathways targeted by increased miR-124 appears as a key factor in the deleterious effects of MDD on brain development. Our main conclusions are summarized in Figure 8.

The direct effects of MDD on the one-carbon metabolism and its various key factors in the animal model as well as the effects of folate deficiency in cultured H19-7 hippocampal progenitors have been extensively described in previous reports. ${ }^{12,14,26}$ In particular, such treatments result in the accumulation of homocysteine, and in a decrease of the cellular index of methylation capacity corresponding to the SAM/SAH ratio. This is reflected in our study notably by a decrease in the amounts of methylated DNA in response to MDD in our two experimental models.

In the in vivo model, it appeared that the deficient regimen provided to dams during gestation led to reduced embryonic growth as compared with gestationally matched control fetuses at E20. These observations are in good accordance with other studies, in which it was shown that embryonic folate concentration limits growth and developmental progression, ${ }^{19}$ whereas B12 deficiency affects body weight of the offspring. ${ }^{33}$ In the present study, such a retardation includes deficiencyassociated reduced brain weight. Decreased proliferation of neuroprogenitors and increased apoptosis have been reported in the brain of rodents under folate and vitamin B12 deficiency during gestation. ${ }^{11,12}$ Importantly, atrophy of various brain layers including hippocampal cell layers and the SVZ - known as neurogenic zones in the adult brain - was 

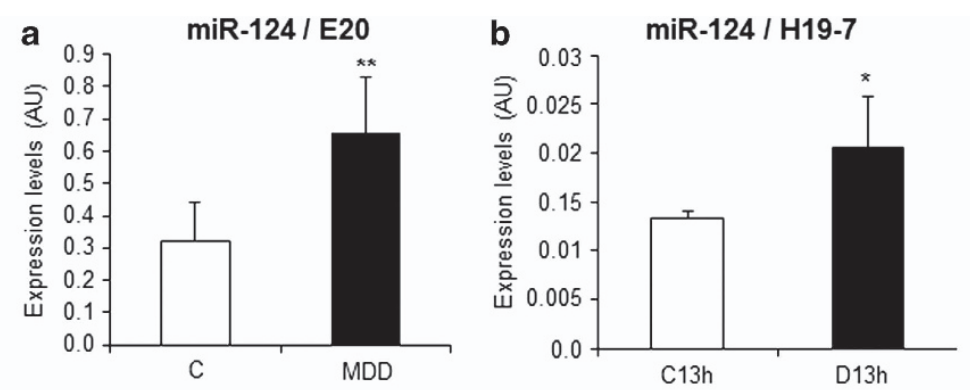

C

miR124 / DAPI
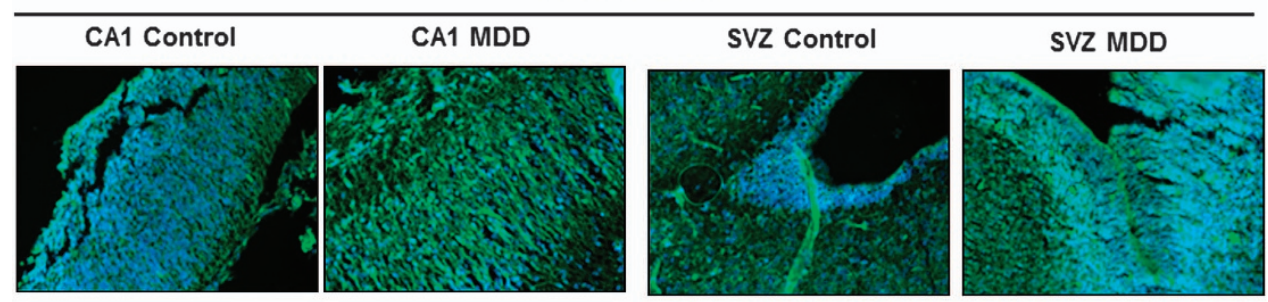

d miR124 I DAPI

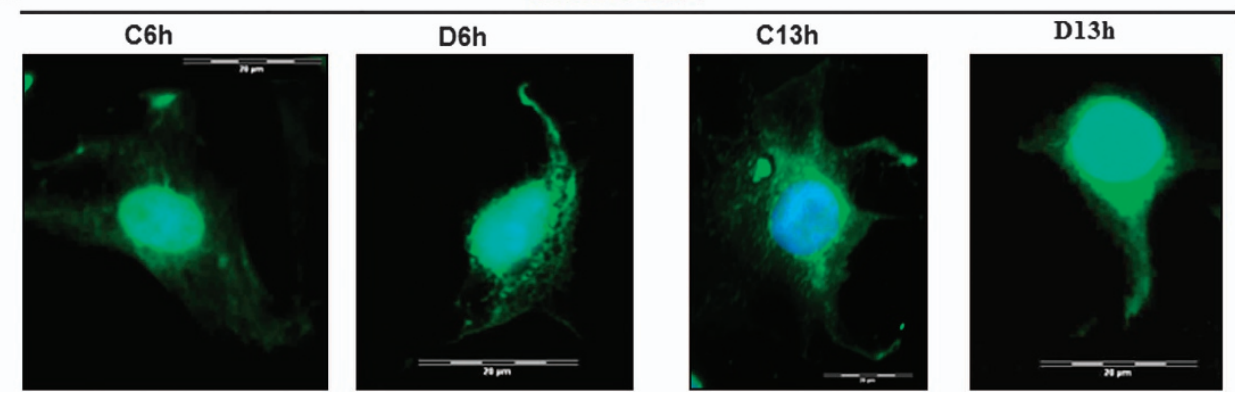

Figure 6 Effects of methyl donor deficiency on the expression of miR-124. (a) Expression levels of miR-124 in AU in the brains of control and deficient E20 fetuses. (b) Expression levels in control (C) and B9-deficient (D) H19-7 cells at $13 \mathrm{~h}$ after the induction of their differentiation. All data are reported as means \pm S.D. ( $n=9$ and $n=7$ for E20 brain tissue and H19-7-cultured cells, respectively), ${ }^{*} P<0.05,{ }^{* *} P<0.01$. (c) Expression of miR-124 as depicted by in situ hybridization in the CA1 layer and SVZ from control and MDD E20 fetuses, and (d) in control (C) and B9-deficient (D) H19-7 cells at 6 and13 h after the induction of their differentiation

depicted in deficient pups. If embryonic growth and brain weight at E20 were reduced in the same proportions (about $15 \%$ ) following exposure to the deficiency, the effects on the cerebral cell layers are more pronounced, especially in the CA1 hippocampal region where pyramidal cell layer was reduced by $40 \%$, reflecting a specific developmental vulnerability under MDD. In addition to decreased cell proliferation, as previously documented, ${ }^{11,14}$ this may be related to increased apoptosis, as observed in situ by the TUNEL assay and caspase labeling, along with the presence of numerous dead cells in the lateral ventricle from deficient fetuses. Cell layer atrophy was still present in the brain of 450-day-old rats, suggesting persisting brain defects.

While folate deprivation markedly affects proliferation, differentiation and viability of hippocampal progenitors in vitro, cell polarity, vesicular transport and synaptic plasticity were shown to be dramatically altered, with poor neurite outgrowth. ${ }^{14}$ The transcription factor Stat3 is known to strongly influence proliferation, survival, growth and differentiation of neuronal cells during brain development and in mature brain after injury. ${ }^{23,34-36}$ In rodents, Stat3 mRNA and protein are expressed continually from E14 to 3 months.
During postnatal development, Stat3 expression increases from postnatal day 3 (PN3) through PN21. ${ }^{23}$ Stat3 acts on various target genes that participate in a variety of physiological processes, including apoptosis, survival and neurite outgrowth. It is activated by phosphorylation at two types of residues, that is, $\mathrm{Tyr}^{705}$ and $\mathrm{Ser}^{727}$, allowing homo- and/or heterodimerization, translocation into the nucleus and subsequent binding to sequence-specific DNA motifs. In addition, phospho-Stat3 Ser ${ }^{727}$ has been recently reported to mediate nerve growth factor-induced neurite outgrowth by a transcription-independent mechanism. ${ }^{37}$ Under MDD, the amounts of Stat3 mRNA, Stat3 and phospho-Stat3 proteins were dramatically decreased both in vivo in the fetal brain and in vitro in hippocampal progenitors, in which less protein amounts were observed in cell nuclei. Stat3 phosphorylation involves various important kinases, the activation of which being differentially affected by methyl donor deprivation. Growth factors such as epidermal growth factor and plateletderived growth factor stimulate Stat tyrosine phosphorylation through their intrinsic receptor tyrosine kinase activity or by the non-receptor tyrosine kinases $\mathrm{Src}^{38}$ whereas serine phosphorylation occurs via MAP kinases and other serine 

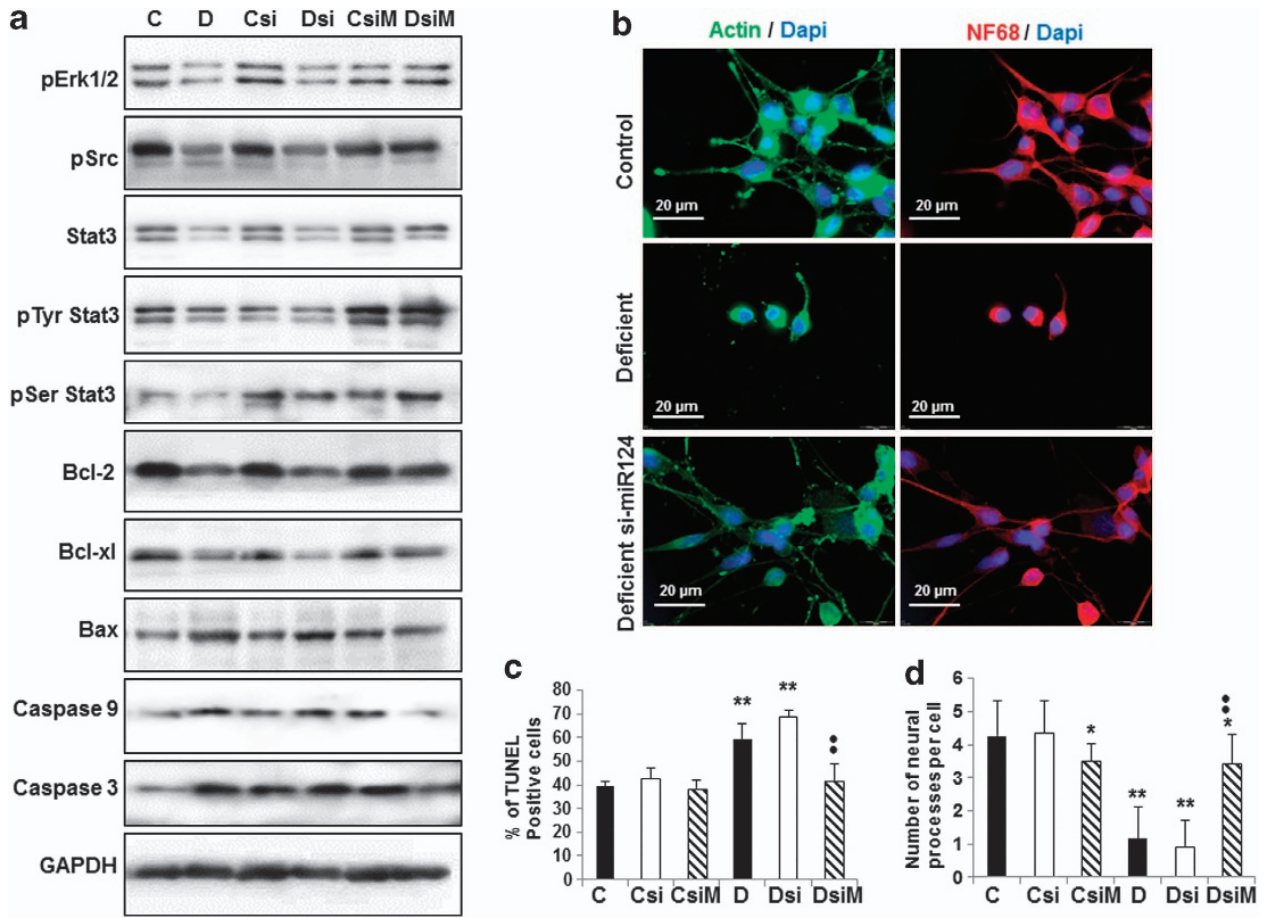

Figure 7 Effects of silencing miR-124 on differentiating H19-7 cells. (a) Effects of miR-124 siRNA on the expression levels of Stat3 and related proteins in control (C) and folate-deficient (D) cells at $13 \mathrm{~h}$ after induction of differentiation ( $\mathrm{si}=$ non-targeting siRNA, siM =miR-124-targeted siRNA). Expression patterns are representative of three separate series of western blots. (b) Influence of miR-124 siRNA on cell morphology at $13 \mathrm{~h}$ after the induction of differentiation. (c) Percentage of apoptotic cells as depicted by the TUNEL assay. (d) Number of cell processes per cell. For (c) and (d): statistically significant difference with the respective control: ${ }^{*} P<0.05$ and ${ }^{* *} P<0.01 ;$ statistically significant difference between Dsi and DsiM: " $P<0.01(n=4)$

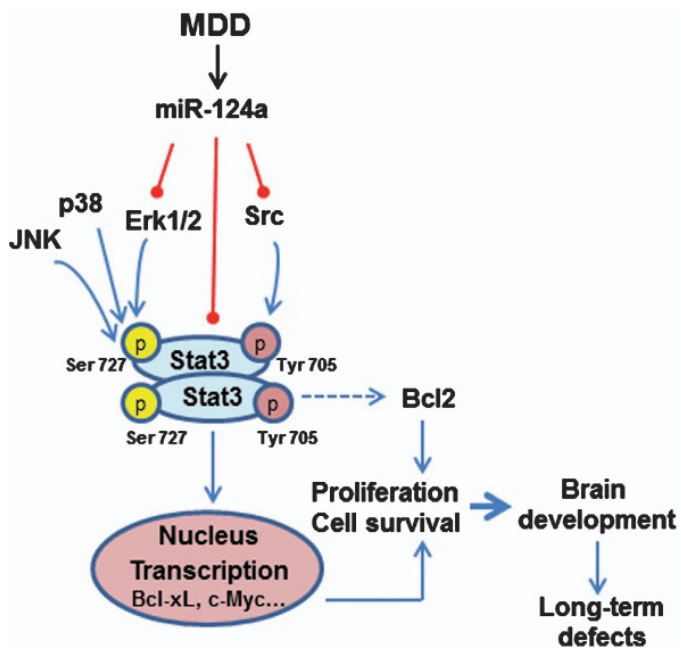

Figure 8 Summary of major events involved in the consequences of MDD in the rat brain. Please note that we provide the first evidence of a negative regulation by miR-124 on Src activity that accounts for phosphorylation of Stat3 at Tyr ${ }^{705}$ residue

kinases such as the serine-threonine kinase Akt. $^{28}$ In our models, phospho-Erk1/2, phospho-Src and phospho-p38 were repressed in conditions of MDD, suggesting their potential role in the pathophysiological consequences observed. Whereas Akt pathway did not appear to be involved, the expression of phospho-JNK was increased, an observation consistent with the potential negative regulation of Stat3 by JNK. ${ }^{39}$

MicroRNAs constitute an extensive class of small noncoding RNAs that control post-transcriptional gene expression. In this respect, Stat3 has been identified as a target of miR-124, the most abundant microRNA in the mammalian brain. The recent identification of a central role for miR-124 in neurogenesis ${ }^{40}$ and synaptic plasticity ${ }^{41}$ integrates this microRNA into the full repertoire of neuronal events, particularly at both the developmental and functional aspects. miR-124 regulates Stat3 either by inhibiting its phosphorylation at $\mathrm{Tyr}^{705}$ residue by an unknown mechanism ${ }^{31}$ or, as demonstrated more recently, by targeting the $3^{\prime}-$ UTR of Stat3 gene so as to repress the expression of Stat3 protein. ${ }^{32}$ The expression levels of miR-124a were found to be obviously increased in brains of fetuses as well as in differentiating hippocampal neurons after exposure to MDD, suggesting a significant role for miR-124 on Stat3 signaling in response to folate and B12 deprivation. This conclusion was strengthened by our additional experiments using siRNA transfection. Indeed, silencing miR-124a by almost $90 \%$ in $\mathrm{H} 19-7$ progenitors appeared to restore Stat3-signaling pathway in folate-deficient cells by increasing specifically the phosphorylation of Erk1/2 and Src kinases. As mentioned above, Erk1/2 phosphorylates Stat3 at Ser ${ }^{727}$ residue, whereas Src phosphorylates Stat3 at $\mathrm{Tyr}^{705}$ residue, the phosphorylation at both residues being necessary for Stat3 activation and 
subsequent dimerization. miR-124 was recently identified as a negative regulator of Erk MAP kinases, ${ }^{42}$ and we provide herein the first evidence of a negative regulation of Src activity by miR-124.

Strikingly, whereas the treatment by miR-124a siRNA slightly reduced the number of neurites in control cells, it greatly improved cell morphology in deficient progenitors.

Although the precise mechanisms by which $B$ vitamin status may influence miR-124 expression remain to be fully elucidated, the existence of a link between folate and miRs has been previously documented. Folate supplementation would exert beneficial effects on ethanol-induced teratogenesis in the fetal mouse brain in part by downregulating miR-10a, ${ }^{43}$ whereas the expression of various miRs in the developing brain of a neural tube defect-prone mutant mouse model (Splotch ${ }^{-1-}$ ) was reversed by maternal folic acid supplementation. ${ }^{44}$ Finally, Marsit et al. ${ }^{45}$ showed that folate deficiency causes a pronounced increase in the expression profiles of several miRs in cultured human lymphoblastoid cells. Reduced folate/vitamin B12 status leads to reduced availability of SAM, which is required for cellular methylation reactions. It has been documented that miRs are regulated by epigenetic events, ${ }^{46}$ and methylation-mediated silencing of the miR-124 genes has been recently demonstrated. ${ }^{47}$ Therefore, altered miR-124 expression may be related to the low methyl donor pool, along with reduced DNA methylation, as observed in our models, and finally resulting in changes in Stat3 expression.

In conclusion, we showed that MDD was associated with an increased miR-124 expression that affected Stat3 signaling at two different levels; first at the expression level by targeting Stat3 mRNA, and second by targeting its activation through the inhibition of Erk1/2 and Src kinases, without effects of miR-124 on other kinases involved in Stat3 signaling. The brain is very vulnerable during development, and impaired Stat3 signaling may lead to reduced cell proliferation and increased apoptosis that may account for a definitive atrophy of specific cerebral layers, as observed in vitamin B-deficient rats.

\footnotetext{
Materials and Methods

Animals and tissue collection. In vivo experiments were conducted in accordance with the NIH Guide for the Care and Use of Laboratory Animals on a validated animal model of MDD, ${ }^{12,17}$ and was approved by the local University Research Ethics Board. One month before mating, adult female Wistar rats (Charles River, l'Arbresle, France) were fed either a standard diet $(n=6)$ (Maintenance diet M20, Scientific Animal Food and Engineering, Villemoissonsur-Orge, France) or a methyl donor-deficient (MDD) low-choline diet lacking folate and vitamin B12 $(n=6)$ (Special Diet Service, Saint-Gratien, France). As gestation progressed, fetuses were collected at E20 from four MDD and four control dams. The fetuses were weighed and evaluated morphologically with the aid of a BX51WI microscope (Olympus, Rungis, France) coupled to an Olympus camera (U-CMAD3). The brains were removed, weighed and conserved for histopathological evaluations. For biochemical analyses, the brains were rapidly harvested and dissected in three parts (cortex, midbrain and cerebellum) before freezing in liquid nitrogen and stored at $-80^{\circ} \mathrm{C}$. Otherwise, the assigned diet was provided to the dams until weaning of the offspring (postnatal day 21). At this time, rat pups were randomly chosen in the different litters (five controls and five MDD), whereas the remaining pups were kept under standard conditions until 450 days of age. In all cases, rats were euthanized by excess isoflurane, and their brains rapidly harvested, frozen in methylbutane at $-30^{\circ} \mathrm{C}$ and stored at $-80^{\circ} \mathrm{C}$ for subsequent immunohistochemistry performed on cryostat-generated $12-\mu \mathrm{m}$ sagittal brain sections mounted onto glass slides.
}

Cell cultures. H19-7/IGF-IR cell line (ATCC no. CRL-2526) was conditionally immortalized from embryonic day 17 rat hippocampi with a temperature-sensitive simian virus 40 (SV40) large tumor antigen. ${ }^{48}$ Cells were seeded at a density of $3 \times 10^{5}$ cells per well in 24-well plates (Falcon, Dutscher, Brumath, France) precoated with poly-L-lysine in high-glucose Dulbecco's modified Eagle's medium (DMEM) supplemented with $10 \%$ fetal calf serum, $50 \mathrm{U} / \mathrm{ml}$ penicillin, $50 \mathrm{U} / \mathrm{ml}$ streptomycin, $2 \mathrm{mM}$ glutamine and $1 \mathrm{mM}$ sodium pyruvate, as previously described. ${ }^{14,49}$ For homogeneity, the same lot of serum was used throughout all experiments. Cells were allowed to proliferate at the temperature of $33^{\circ} \mathrm{C}$ in $95 \%$ air $/ 5 \% \mathrm{CO}_{2}$. Selection was maintained with $200 \mu \mathrm{g} / \mathrm{ml} \mathrm{G} 418$. As standard DMEM does not contain vitamin B12, MDD was induced by using DMEM lacking vitamin B9 (Invitrogen, Cergy-Pontoise, France). After $24 \mathrm{~h}$, cells were washed and shifted to the non-permissive temperature $\left(39^{\circ} \mathrm{C}\right)$ for induction of their differentiation in high-glucose-DMEM (with or without B9) containing $1 \%$ fetal calf serum, $\mathrm{N}_{2}$ supplement (Invitrogen) and $0.11 \mathrm{mg} / \mathrm{ml}$ sodium pyruvate, $2 \mathrm{mM}$ glutamine, $0.1 \mathrm{mg} / \mathrm{ml}$ transferrin, $20 \mathrm{nM}$ progesterone, $0.1 \mathrm{mM}$ putrescine and $30 \mathrm{nM}$ sodium selenite.

Cell viability was assessed by the measurement of intracellular ATP using the CellTiter-Glo luminescent viability assay (Promega, Charbonnières-les-Bains, France), and mitochondrial membrane permeability was evaluated by the MitoCapture assay kit (Calbiochem, VWR International, SAS, Fontenay-sous-Bois, France) using a fluorescent cationic dye, as described by Akchiche et al. ${ }^{49}$

DNA methylation. Global DNA methylation was quantified in brain tissues at E20 as well as in cultured hippocampal cells at $13 \mathrm{~h}$ after the induction of differentiation by using the Methylamp Global DNA Methylation Quantification Kit (Epigentek, Euromedex, Mundolsheim, France), as previously described. ${ }^{50}$ According to the manufacturer's instructions, the methylated fraction of DNA can be recognized by a 5-methylcytosine antibody and quantified through an ELISA-like reaction. The amount of methylated DNA is proportional to the OD intensity.

Histopathological analyses. For basic histopathological investigations, brain sections were stained with thionin or the DNA fluorochrome 4,6-diamidino-2phenylindole (Dapi, Sigma-Aldrich, Saint-Quentin Fallavier, France) for the measurement of thickness of specific brain layers.

The presence of apoptosis was selectively depicted by the Apostain method ${ }^{51}$ using monoclonal antibody to single-strand DNA (F7-26 from calf thymus ssDNA, AbCys SA, Paris, France) after DNA denaturation by heating in the presence of formamide, as described by Blaise et al. ${ }^{12}$ and Akchiche et al. ${ }^{14}$ for tissue sections and cultured cells, respectively. Apoptosis was additionally monitored by using the ApopTag Plus Fluorescein In Situ Apoptosis Detection Kit (Chemicon International, Millipore, Guyancourt, France) based on the TUNEL assay and which labels apoptotic cells by modifying genomic DNA utilizing terminal deoxynucleotidyl transferase (TdT).

For skeletal analysis, the procedure adapted from Wallin et al. ${ }^{52}$ involves complete skinning of fresh eviscerated rat embryos following a 24 -h-immersion in $95 \%$ ethanol. Skeletons were stained for $48 \mathrm{~h}$ with $1 \%$ Alcian blue dye, diluted in an ethanol/acetic acid mix, specific for cartilage staining. Skeletons were then macerated in $2 \% \mathrm{KOH}$ until bones are visible, and stained for $24 \mathrm{~h}$ with $0.12 \%$ Alizarin red dye diluted in $\mathrm{KOH}$, specific for bone staining. Specimen were finally cleared and hardened in glycerol/ethanol baths and stored in $87 \%$ glycerol. Femur length was measured by means of the Cell software (Olympus).

Immunohistochemistry. Cells cultured on poly-L-lysine-precoated glass coverslips were washed, fixed with $4 \%$ paraformaldehyde and permeabilized with $0.1 \%$ Triton X-100 (Sigma-Aldrich), as reported by Akchiche et al. ${ }^{14}$ Nonspecific binding sites were blocked in phosphate-buffered saline containing $1 \%$ bovine serum albumin (BSA) and incubation was performed overnight with an antibody against one of the following proteins: Stat3 (rabbit monoclonal, 1/200, Cell Signaling Technology, Ozyme, Saint-Quentin en Yvelines, France), phospho-Stat3 Tyr $^{705}$ (mouse monoclonal, 1/100, Cell Signaling Technology), phospho-Stat3 $\mathrm{Ser}^{727}$ (mouse monoclonal, 1/100, Enzo life sciences, Villeurbanne, France), actin (goat polyclonal, 1/200, Santa Cruz Biotechnology, Tebu-Bio, Le Perrayen-Yvelines, France), NF68 (rabbit polyclonal, 1/300, US Biological, Euromedex, Souffelweyersheim, France). After a washing step, immunoreactivity was assessed by incubation in the presence of an appropriate secondary anti-lgG antibody conjugated to AlexaFluor for $1 \mathrm{~h}$ at $25^{\circ} \mathrm{C}(1 / 1000$, Molecular Probes, Cergy-Pontoise, France). Control experiments were conducted by omitting the primary antibody. Immunofluorescence visualization, image acquisition $(\times 20$ and 
$\times 60$ magnification) and unbiased cell counts in randomly selected fields were performed with a BX51WI microscope (Olympus) coupled to an Olympus camera (U-CMAD3) or a confocal microscope (Nikon Instruments, Champigny sur Marne Cedex, France), and analyzed using Cell software. Labeled cells were calculated as a percent of total cells after Dapi counterstaining.

Western blotting. Western blot analyses were performed using standard procedure with chemiluminescence using ECL system (Amersham Biosciences, Orsay, France), as previously detailed. ${ }^{49}$ Antibodies against the following proteins were used: Stat3 (rabbit monoclonal, 1/1000, Cell Signaling Technology), phospho-Stat3 Tyr ${ }^{705}$ (mouse monoclonal, 1/1000, Cell Signaling Technology), phospho-Stat3 $\mathrm{Ser}^{727}$ (mouse monoclonal, 1/250, Enzo life sciences), Bcl-2 (goat polyclonal, 1/1000, Santa Cruz Biotechnology), Bcl-xL (rabbit polyclonal, 1/1000, Cell Signaling Technology), Bax (rabbit polyclonal, 1/1000, Santa Cruz Biotechnology), cleaved caspase-3, Asp ${ }^{175}$ (rabbit polyclonal, 1/1000, Cell Signaling Technology), cleaved caspase-9, Asp ${ }^{353}$ (rabbit polyclonal, 1/1000, Cell Signaling Technology), Src (rabbit polyclonal, 1/500, Cell Signaling Technology), phospho-Src Family Tyr ${ }^{416}$ (rabbit monoclonal, 1/1000, Cell Signaling Technology), p44/42 MAP kinase (Erk1/2, rabbit polyclonal, 1/500, Cell Signaling Technology), phospho-p44/42 MAP kinase $\mathrm{Thr}^{202} / \mathrm{Tyr}^{204}$ (rabbit polyclonal, $1 / 500$, Cell Signaling Technology), Akt (rabbit polyclonal, 1/1000, Cell Signaling Technology), phospho-Akt Ser ${ }^{73}$ (rabbit polyclonal, 1/1000, Cell Signaling Technology), phospho-p38 MAP kinase $\mathrm{Thr}^{180} / \mathrm{Tyr}^{182}$ (rabbit monoclonal, 1/500, Cell Signaling Technology) and phospho-SAPK/JNK $\mathrm{Thr}^{183} / \mathrm{Tyr}^{185}$ (rabbit monoclonal, 1/500, Cell Signaling Technology).

Glyceraldehyde-3-phosphate dehydrogenase (GAPDH, mouse monoclonal, $1 / 1000$, Abcam, Paris, France) was used as an internal standard. Polyvinylidene difluoride membranes were incubated for $1 \mathrm{~h}$ at room temperature with the corresponding horseradish peroxidase-conjugated preadsorbed secondary antibody (1/5000, Molecular Probes). Quantity One software, associated with the VersaDoc imaging system (Model 1000, Bio-Rad Laboratories, Marnes-la-Coquette, France), was used to quantify signals.

RNA extraction. Total RNA was extracted from H19-7 cells $\left(10^{2}-10^{7}\right.$ cells) and from $0.5 \mathrm{mg}$ of embryonic midbrain tissues using the mirVana miRNA Isolation kit (Applied Biosystems, Foster City, CA, USA) following the manufacturer's instructions. miRNA is isolated using a two-step procedure. In the first step, samples are disrupted in a denaturing lysis buffer, and then subjected to acidphenol/chloroform extraction. The second step consists of purification over glassfiber filter that immobilizes the RNA that was later eluted using RNase-free water. According to the manufacturer's instructions, no enrichment procedure is needed while isolating miRNA for expression profiling using miRNA arrays. The concentration and purity of RNA were determined at $260 / 280 \mathrm{~nm}$ by using a nanodrop spectrophotometer (Multiskan GO, Thermo Scientific, Gometz le Châtel, France).

Quantitative real-time RT-PCR. Total RNA was purified using the RNeasy Plus Mini kit (Qiagen). PCR was performed using the Quantitect SYBR Green PCR kit from Qiagen and the LightCycler 480 instrument (Roche Applied Science, Manheim, Germany). Specific amplifications were performed using as primers: Stat3 forward 5'-GAAGAGTGCCTTCGTGGT-3', reverse 5'-AGCAACCT GACTTTTGTGG-3'. Quantification was performed with RNA polymerase ॥ (Pol II) as an internal standard with the following primers: forward $5^{\prime}$-GCATTAACAT CAGGAACAATAAAGGC- $3^{\prime}$ and reverse $5^{\prime}$-GATCTCTCTAAAGTTGACCT CATTGG-3'. Real-time PCR was carried out using the DNA-binding dye SYBR Green I for the detection of PCR products. Temperature cycling was $15 \mathrm{~min}$ at $95^{\circ} \mathrm{C}$ followed by 50 cycles consisting of $94^{\circ} \mathrm{C}$ for $10 \mathrm{~s}, 56^{\circ} \mathrm{C}$ for $15 \mathrm{~s}$ and $72{ }^{\circ} \mathrm{C}$ for $15 \mathrm{~s}$. Results were expressed as arbitrary units by calculating the ratio of crossing points of amplification curves stat3 mRNAs and internal standard by using the RelQuant software (Roche Diagnostics).

\section{Analysis of miR-124 expression}

TaqMan RT-qPCR: Two-step real-time PCR was used to analyze the expression of miR-124. In the first step, total RNA was reverse transcribed using miRNA-specific RT primers (rno-miR-124 and U6SnoRNA) and a TaqMan MicroRNA Reverse Transcription Kit (Applied Biosystems). miRNA expression was analyzed using Taqman microRNA assays (Applied Biosystems), according to the instructions of the manufacturer. The RT reaction was performed in $15 \mu$ l volume, containing $1 \mu \mathrm{g}$ RNA sample, $3 \mu \mathrm{l}$ primer and master mix adjusted to $15 \mu \mathrm{l} /$ reaction. Products of $\mathrm{RT}$ reaction $(1.33 \mu \mathrm{l})$ were used in a real-time PCR reaction, which also included $10 \mu \mathrm{l}$ of the TaqMan Universal Master Mix II, and $1 \mu \mathrm{l}$ TaqMan miRNA assay containing the sequence-specific primers of either the target miRNA (5'-GGCATTCACCGCGTGCCTTA-3') or the U6SnoRNA (5'-CACGAATTTGCGTGTCATCCTT- $3^{\prime}$ ) used as an endogenous control for normalization. Real-time PCR was carried out on a Step One Plus LightCycler (Roche Applied Science). Incubations were performed in a 96 -well plate at $95^{\circ} \mathrm{C}$ for $10 \mathrm{~min}$ for enzyme activation, followed by 40 cycles of PCR: denaturation $\left(95^{\circ} \mathrm{C}\right.$ for $\left.15 \mathrm{~s}\right)$, and annealing/extending $\left(60^{\circ} \mathrm{C}\right.$ for $\left.2 \mathrm{~min}\right)$. Data analysis was performed with the software provided by the manufacturer (Step One Plus).

In situ hybridization: The in situ detection of miR-124 was performed on paraffin-embedded sections from normal and methyl donor-deficient brain tissues by locked nucleic acid (LNA)-oligo in situ hybridization, as previously described by Kloosterman et al. ${ }^{53}$ Briefly, the slides were deparaffinized in xylene, rehydrated in decreasing concentrations of ethanol and treated with proteinase-k for nucleic acid release. Slides were then re-dehydrated and prehybridized in hybridization buffer with $0.5 \mathrm{~nm}$ specific probe (LNA-modified and digoxygenin (DIG)-labeled oligonucleotide, Exiqon, Copenhagen, Denmark) complementary to miR-124. Sections were then washed in saline sodium citrate buffer, followed by blocking in Denhardt solution $1 \times$ in a humidified chamber. Slides were then incubated with anti-DIG antibody (1/500, Roche Applied Science) for $1 \mathrm{~h}$ at room temperature, washed in PBS-T and then immunoreactivity was assessed in the presence of a matching secondary antibody conjugated to AlexaFluor (1/2000, Molecular probes) for $1 \mathrm{~h}$ at room temperature. Positive controls (snoRNA U6B, Exiqon) were used for each hybridization experiment.

Small interfering RNA (siRNA) and cell transfection. MiR-124 expression in hippocampal cell cultures was silenced using small interfering RNA (siRNA). The siRNA oligonucleotide duplexes were purchased from Ambion (Applied Biosystems) for targeting the rat miR-124 (has-miR-124-3p) in H19-7 cells. The siRNA sequence is (sense strand-indicated): $5^{\prime}$-UAAGGCACGCGGU GAAUGCC- $3^{\prime}$, and mirVana miRNA Inhibitor Negative Control no. 1 was used as control for evaluation of the effect of the experimental miRNA inhibition. siRNA duplexes $(25 \mathrm{nM}$ final concentration for the first transfection then $10 \mathrm{nM}$ for the second day re-transfection) were transfected with lipofectamine RNAiMax (Invitrogen). Three days post transfection, cells were harvested for RNA or protein extraction.

Statistical analysis. Data were analyzed with Statview 5 software for Windows (SAS Institute, Berkley, CA, USA). They were compared by using one-way analysis of variance (ANOVA) with Fisher's test. $P$-value $<0.05$ was considered to indicate significance.

\section{Conflict of Interest}

The authors declare no conflict of interest.

Acknowledgements. We are indebted to the 'Région Lorraine' for support. RK, $A G$ and $A B$ are recipients of a fellowship from the french Ministry for Higher Education and Research.

1. Oliver MH, Jaquiery AL, Bloomfield FH, Harding JE. The effects of maternal nutrition around the time of conception on the health of the offspring. Soc Reprod Fertil Suppl 2007; 64: $397-410$.

2. McMillen IC, MacLaughlin SM, Muhlhausler BS, Gentili S, Duffield JL, Morrison JL. Developmental origins of adult health and disease: the role of periconceptional and foetal nutrition. Basic Clin Pharmacol Toxicol 2008; 102: 82-89.

3. Attig L, Gabory A, Junien C. Nutritional developmental epigenomics: immediate and longlasting effects. Proc Nutr Soc 2010; 69: 221-231.

4. Amouzou EK, Chabi NW, Adjalla CE, Rodriguez-Guéant RM, Feillet F, Villaume $\mathrm{C}$ et al. High prevalence of hyperhomocysteinemia related to folate deficiency and the $677 \mathrm{C}->\mathrm{T}$ mutation of the gene encoding methylenetetrahydrofolate reductase in coastal West Africa. Am J Clin Nutr 2004; 79: 619-624.

5. McLean E, de Benoist B, Allen LH. Review of the magnitude of folate and vitamin B12 deficiencies worldwide. Food Nutr Bull 2008; 29: S38-S51.

6. Guéant JL, Namour F, Guéant-Rodriguez RM, Daval JL. Folate and fetal programming: a play in epigenomics? Trends Endocrinol Metab 2013; 24: 279-289. 
7. Finkelstein JD. The metabolism of homocysteine: pathways and regulation. Eur J Pediatr 1998; 157(Suppl 2): S40-S44.

8. Molloy AM, Kirke PN, Brody LC, Scott JM, Mills JL. Effects of folate and vitamin B12 deficiencies during pregnancy on fetal, infant, and child development. Food Nutr Bull 2008; 29: S101-S111

9. Black MM. Effects of vitamin B12 and folate deficiency on brain development in children Food Nutr Bull 2008; 29: S126-S131.

10. Kirke PN, Molloy AM, Daly LE, Burke H, Weir DG, Scott JM. Maternal plasma folate and vitamin B12 are independent risk factors for neural tube defects. Q J Med 1993; 86: 703-708.

11. Craciunescu CN, Brown EC, Mar MH, Albright CD, Nadeau MR, Zeisel SH. Folic acid deficiency during late gestation decreases progenitor cell proliferation and increases apoptosis in fetal mouse brain. J Nutr 2004; 134: 162-166.

12. Blaise SA, Nédélec E, Schroeder H, Alberto JM, Bossenmeyer-Pourié C, Guéant JL et al. Gestational vitamin B deficiency leads to homocysteine-associated brain apoptosis and alters neurobehavioral development in rats. Am J Pathol 2007; 170: 667-679.

13. Battaglia-Hsu SF, Akchiche N, Noel N, Alberto JM, Jeannesson E, Orozco-Barrios CE et al. Vitamin $B 12$ deficiency reduces proliferation and promotes differentiation of neuroblastoma cells and up-regulates PP2A, proNGF, and TACE. Proc Natl Acad Sci USA 2009; 106 21930-21935.

14. Akchiche N, Bossenmeyer-Pourié C, Kerek R, Martin N, Pourié G, Koziel V et al Homocysteinylation of neuronal proteins contributes to folate deficiency-associated alterations of differentiation, vesicular transport, and plasticity in hippocampal neuronal cells. FASEB J 2012; 26: 3980-3992.

15. Ghemrawi R, Pooya S, Lorentz S, Gauchotte G, Arnold C, Gueant JL et al. Decreased vitamin $B 12$ availability induces ER stress through impaired SIRT1-deacetylation of HSF1. Cell Death Dis 2013; 4: e553.

16. Troen AM, Shea-Budgell M, Shukitt-Hale B, Smith DE, Selhub J, Rosenberg IH. B-vitamin deficiency causes hyperhomocysteinemia and vascular cognitive impairment in mice. Proc Natl Acad Sci USA 2008; 105: 12474-12479.

17. Daval JL, Blaise S, Guéant JL. Vitamin B deficiency causes neural cell loss and cognitive impairment in the developing rat. Proc Natl Acad Sci USA 2009; 106: E1.

18. Greene ND, Dunlevy LE, Copp AJ. Homocysteine is embryotoxic but does not cause neura tube defects in mouse embryos. Anat Embryol (Berl) 2003; 206: 185-191.

19. Burren KA, Savery D, Massa V, Kok RM, Scott JM, Blom HJ et al. Gene-environment interactions in the causation of neural tube defects: folate deficiency increase susceptibility conferred by loss of Pax3 function. Hum Mol Genet 2008; 17: 3675-3685.

20. Greene ND, Copp AJ. Development of the vertebrate central nervous system: formation of the neural tube. Prenat Diagn 2009; 29: 303-311.

21. Levy DE, Lee CK. What does Stat3 do? J Clin Invest 2002; 109: 1143-1148.

22. Ihle JN. The Stat family in cytokine signaling. Curr Opin Cell Biol 1993; 13: 211-217.

23. Dziennis S, Alkayed NJ. Role of signal transducer and activator of transcription 3 in neuronal survival and regeneration. Rev Neurosci 2008; 19: 341-361.

24. Conway G. STAT3-dependent pathfinding and control of axonal branching and target selection. Dev Biol 2006; 296: 119-136.

25. He JC, Gomes I, Nguyen T, Jayaram G, Ram PT, Devi LA et al. The G alpha(o/i)-coupled cannabinoid receptor-mediated neurite outgrowth involves Rap regulation of $\mathrm{Src}$ and Stat3. $J$ Biol Chem 2005; 280: 33426-33434.

26. Blaise SA, Nédélec E, Alberto JM, Schroeder H, Audonnet S, Bossenmeyer-Pourié C et al. Short hypoxia could attenuate the adverse effects of hyperhomocysteinemia on the developing rat brain by inducing neurogenesis. Exp Neurol 2009; 216: 231-238.

27. Turkson J, Bowman T, Garcia R, Caldenhoven E, De Groot RP, Jove R. Stat3 activation by Src induces specific gene regulation and is required for cell transformation. Mol Cell Biol 1998; 18: 2545-2552.

28. Decker T, Kovarik P. Serine phosphorylation of STATs. Oncogene 2000; 19 2628-2637.

29. Rahaman SO, Harbor PC, Chernova O, Barnett GH, Vogelbaum MA, Haque SJ. Inhibition of constitutively active Stat3 suppresses proliferation and induces apoptosis in glioblastoma multiforme cells. Oncogene 2002; 21: 8404-8413.

30. Choi H J, Lee J H, Park SY, Cho JH, Han JS. STAT3 is involved in phosphatidic acidinduced Bcl-2 expression in HeLa cells. Exp Mol Med 2009; 41: 94-101.

31. Krichevsky AM, Sonntag KC, Isacson O, Kosik KS. Specific microRNAs modulate embryonic stem cell-derived neurogenesis. Stem Cells 2006; 24: 857-864.
32. Cai B, Li J, Wang J, Luo X, Ai J, Liu Y et al. microRNA-124 regulates cardiomyocyte differentiation of bone marrow-derived mesenchymal stem cells via targeting STAT3 signaling. Stem Cells 2012; 30: 1746-1755.

33. Molina V, Medici M, Taranto MP, Font de Valdez G. Effects of maternal vitamin B12 deficiency from end of gestation to weaning on the growth and haematological and immunological parameters in mouse dams and offspring. Arch Anim Nutr 2008; 62: 162-168.

34. Deverman BE, Patterson PH. Cytokines and CNS development. Neuron 2009; 64: 61-78.

35. Cheng X, Jin G, Zhang X, Tian M, Zou L. Stage-dependent STAT3 activation is involved in the differentiation of rat hippocampus neural stem cells. Neurosci Lett 2011; 493: 18-23.

36. Murase S, Kim E, Lin L, Hoffman DA, McKay RD. Loss of signal transducer and activator of transcription 3 (STAT3) signaling during elevated activity causes vulnerability in hippocampal neurons. J Neurosci 2012; 32: 15511-15520.

37. Zhou L, Too HP. Mitochondrial localized STAT3 is involved in NGF induced neurite outgrowth. PLoS One 2011; 6: e21680.

38. Zorina $\mathrm{Y}$, lyengar R, Bromberg KD. Cannabinoid 1 receptor and interleukin-6 receptor together induce integration of protein kinase and transcription factor signaling to trigger neurite outgrowth. J Biol Chem 2010; 285: 1358-1370.

39. Lim CP, Cao X. Serine phosphorylation and negative regulation of Stat3 by JNK. J Biol Chem 1999; 274: 31055-31061.

40. Akerblom M, Sachdeva R, Barde I, Verp S, Gentner B, Trono D et al. MicroRNA-124 is a subventricular zone neuronal fate determinant. J Neurosci 2012; 32: 8879-8889.

41. Rajasethupathy P, Fiumara F, Sheridan R, Betel D, Puthanveettil SV, Russo JJ et al. Characterization of small RNAs in aplysia reveals a role for miR-124 in constraining synaptic plasticity through CREB. Neuron 2009; 63: 803-817.

42. Yamane K, Jinnin M, Etoh T, Kobayashi Y, Shimozono N, Fukushima S et al. Downregulation of miR-124/-214 in cutaneous squamous cell carcinoma mediates abnormal cell proliferation via the induction of ERK. J Mol Med (Berl) 2013; 91: 69-81.

43. Wang LL, Zhang Z, Li Q, Yang R, Pei X, Xu Y et al. Ethanol exposure induces differential microRNA and target gene expression and teratogenic effects which can be suppressed by folic acid supplementation. Hum Reprod 2009; 24: 562-579.

44. Ichi S, Costa FF, Bisch of JM, Nakazaki H, Shen YW, Boshnjaku V et al. Folic acid remodels chromatin on Hes1 and Neurog2 promoters during caudal neural tube development. J Biol Chem 2010; 285: 36922-36932.

45. Marsit CJ, Eddy K, Kelsey KT. MicroRNA responses to cellular stress. Cancer Res 2006; 66: 10843-10848.

46. Sato F, Tsuchiya S, Meltzer SJ, Shimizu K. MicroRNAs and epigenetics. FEBS J 2011; 278: 1598-1609.

47. Wang $\mathrm{P}$, Chen L, Zhang J, Chen H, Fan J, Wang K et al. Methylation-mediated silencing of the miR-124 genes facilitates pancreatic cancer progression and metastasis by targeting Rac1. Oncogene 2013; e-pub ahead of print 21 January 2013; doi:10.1038/onc.2012.598.

48. Eves EM, Tucker MS, Roback JD, Downen M, Rosne MR, Wainer BH. Immortal rat hippocampal cell lines exhibit neuronal and glial lineages and neurotrophin gene expression. Proc Natl Acad Sci USA 1992; 89: 4373-4377.

49. Akchiche N, Bossenmeyer-Pourié C, Pourié G, Koziel V, Nédélec E, Guéant JL et al. Differentiation and neural integration of hippocampal neuronal progenitors: signaling pathways sequentially involved. Hippocampus 2010; 20: 949-961.

50. Wang Bx Yin BL, He B, Chen C, Zhao M, Zhang Wx et al. Overexpression of DNA damageinduced $45 \alpha$ gene contributes to esophageal squamous cell cancer by promoter hypomethylation. J Exp Clin Cancer Res 2012; 31: 11.

51. Frankfurt OS, Krishan A. Identification of apoptotic cells by formamide-induced dna denaturation in condensed chromatin. J Histochem Cytochem 2001; 49: 369-378.

52. Wallin J, Wilting J, Koseki H, Fritsch R, Christ B, Balling R. The role of Pax-1 in axial skeleton development. Development 1994; 120: 1109-1121.

53. Kloosterman WP, Wienholds E, de Bruijn E, Kauppinen S, Plasterk RH. In situ detection of miRNAs in animal embryos using LNA-modified oligonucleotide probes. Nat Methods 2006; 3: 27-29.

Cell Death and Disease is an open-access journal con
licensed under a Creative Commons Attribution-NonCommercialNoDerivs 3.0 Unported License. To view a copy of this license, visit http://creativecommons.org/licenses/by-nc-nd/3.0/ 